\title{
Stability analysis of an insulationless HTS pancake coil under the magnitude of external magnetic field
}

\author{
Sung-Jun Jung, Gyeong-Hun Kim, Kwangmin Kim, Minwon Park and In-Keun Yu* \\ Changwon National University, Sarim-dong, Changwon, 641-773, Korea
}

Received 05 October 2012; accepted 20 November 2012

\begin{abstract}
An HTS (high temperature superconducting) coil without insulation has been investigated since a metallic insulation was suggested in the mid-1980s. The advantage of an insulationless HTS pancake coil is that it is more stable than an insulated HTS pancake coil. This paper focuses on the various characteristics of the insulationless HTS pancake coil related with stability, especially under the external magnetic field. Because HTS pancake coil may be influenced by the external magnetic field in a real operational environment of electrical devices. First, charge-discharge test was performed for the characteristics evaluation of the insulationless HTS pancake coil as compared with insulated HTS pancake coil in liquid nitrogen at $77 \mathrm{~K}$. Based on the experiment results, characteristics evaluation of the insulationless HTS pancake coil was implemented under the external magnetic field. In order to carry out the experiment, we have fabricated a cylindrical solenoid coil to apply the magnetic field. The various characteristics of the insulationless HTS pancake coil were evaluated for charge-discharge and over-current conditions. This paper proves that the insulationless HTS pancake coil is more stable than the insulated HTS pancake under the external magnetic field.
\end{abstract}

Keywords: External magnetic field, High Temperature superconductor, HTS pancake coil, Insualtionless superconducting coil

\section{INTRODUCTION}

In recent years, superconductivity has been contributed to many different advanced technologies. Insulation, which is an essential element in the superconducting magnet, has been developed with advancement of the superconductivity. Development of insulation by using diverse materials is being rapidly achieved in order to increase dielectric strength and decrease volume of insulation [1]. The superconducting coil without insulation has also been studied since a metallic insulation was suggested by Bailey [2]. The insulationless HTS pancake coil is the HTS pancake coil which removes the electric insulation or inserts metallic insulation between the superconducting layers. The advantages of insulationless HTS pancake coil are as follows, compared with insulated HTS pancake coil. First, there is none of the damage from quench because the coil itself has a very high stability [3, 4]. Second, in the insulationless HTS pancake coil, the

*Corresponding author : yuik@changwon.ac.kr production costs and installation space can be reduced because the protection device that has been applied to the existing superconducting application can decrease. Third, winding is easier than existing insulated pancake coil because the insulation layer between turn and turn is not inserted separately. Therefore the elimination of insulation has many kinds of benefits, particularly its stability.

In this paper, the experiment was performed on the insulated and insulationless HTS pancake coils in liquid nitrogen at $77 \mathrm{~K}$. Specific aspects of each HTS pancake coil were investigated under charge-discharge state. Based on these data, the characteristics evaluation of the insulationless HTS pancake coil was implemented under an external magnetic field, which is dealt with in conventional papers. In order to carry out the experiment, a cylindrical solenoid coil was fabricated using copper wire. Also, the external magnetic field from $0.02 \mathrm{~T}$ to $0.12 \mathrm{~T}$ was applied to HTS coils in parallel and perpendicular magnetic direction. The properties of the insulationless HTS pancake coil varied according to the magnitude of the external magnetic field, because HTS pancake coils may be influenced by the external magnetic field in a real operational environment of electrical devices [5-7]. The critical current of the insulated HTS pancake coil under the external magnetic field decreases and leads to damage. But although the critical current of the insulationless HTS pancake coil also decreases, the insulationless HTS pancake coil didn't damaged from quench due to the current-sharing.

\section{EXPERIMETAL SETUP}

\subsection{Configuration of experiment devices}

Fig. 1 shows the basic connection scheme of experiment devices. A DC power supply was used to apply the current to the external magnet field and an AC power supply was used to apply the current to the HTS pancake coils. SCXI connected with the LabVIEW was used for current control and data measurement. A hall senor was placed on a separate plate and positioned at the center point of each coil to confirm the charge and discharge of the HTS pancake coils. The HTS pancake coil was placed in the center of the cylindrical solenoid coil respectively. All tests were performed in liquid nitrogen at $77 \mathrm{~K}$. Fig. 2 shows the hardware configuration of the experiment devices. 


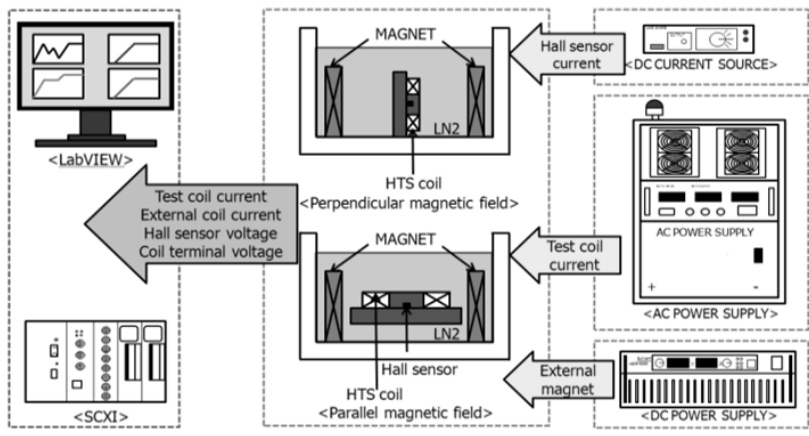

Fig. 1. Basic connection scheme of experiment devices.

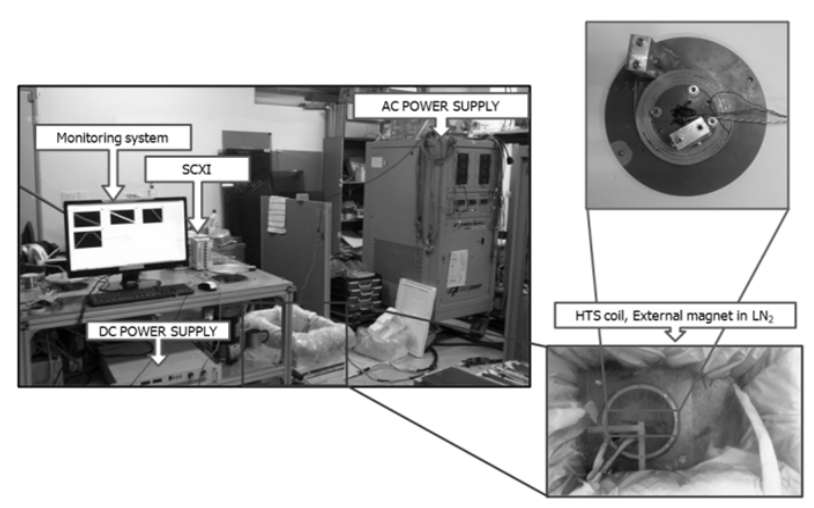

Fig. 2. Hardware configuration of the experiment devices.

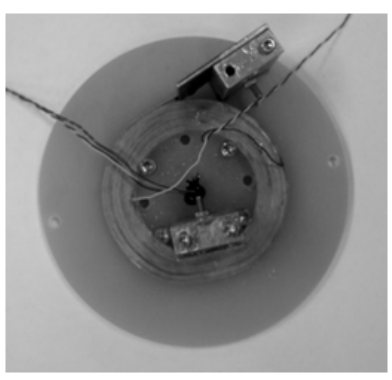

(a)

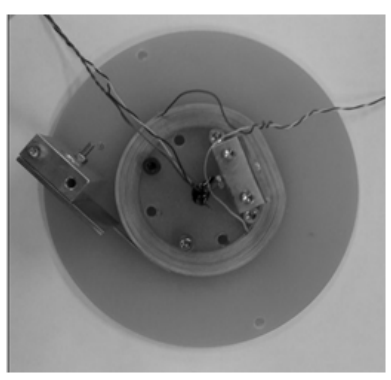

(b)
Fig. 3. (a) Insulated HTS pancake coil, (b) Insulationless HTS pancake coil.

Table I

SPECIFICATIONS OF DESIGNED HTS COILS.

\begin{tabular}{lcc}
\hline \multicolumn{1}{c}{ Parameters } & insulated & insulationless \\
\hline Wire type & BSCCO Wire \\
Wire IC@77 K [A] & \multicolumn{2}{c}{65} \\
Wire width; Thickness [mm] & \multicolumn{2}{c}{$4.43 ; 0.23$} \\
Inner radius of coil [mm] & \multicolumn{2}{c}{25} \\
Outer radius of coil [mm] & 35.35 & 35.8 \\
Total wire length of coil [m] & \multicolumn{2}{c}{8.3} \\
Insulation & Kapton tape & N/A \\
Number of turn & \multicolumn{2}{c}{45} \\
Quench voltage [mV] & \multicolumn{2}{c}{0.83} \\
\hline
\end{tabular}

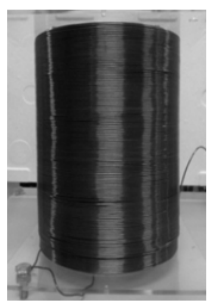

(a)

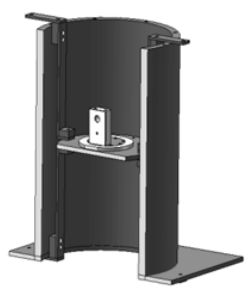

(b)

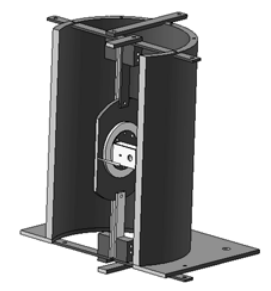

(c)
Fig. 4. (a) External magnet for experiment. (b) Applied to parallel magnetic field. (c) Applied to perpendicular magnetic field.

Table II

Specifications of designed external magnet

\begin{tabular}{lc}
\hline \multicolumn{1}{c}{ Items } & Values \\
\hline Wire type & copper wire \\
Transport current $[\mathrm{A}]$ & 50 \\
Wire diameter [mm] & 2 \\
Inner, Outer radius of coil [mm] & $90 ; 105$ \\
Total wire length of coil [m] & 283 \\
Number of turn, layer & 120,4 \\
magnetic field of center $[\mathrm{T}]$ & 0.1 \\
\hline
\end{tabular}

Table I summarizes the parameters of the HTS pancake coils for the experiment. Both test coils were fabricated with BSCCO wire and the number of turns of the insulated HTS pancake coil and the insulationless HTS pancake coil is 45 . Kapton tape was used for the insulation of the insulated HTS pancake coil. The critical current of the insulated and insulationless HTS pancake coils were $23 \mathrm{~A}$. Fig. 3 shows the HTS pancake test coils for experiment.

\subsection{Fabrication of an external magnet}

Fig. 4 shows the shape of an external field magnet and applied directions of the external magnet. The cylindrical solenoid coil was fabricated by using $2 \mathrm{~mm}$ copper wire to apply the external magnetic field. When the operating current of the external magnetic field is $50 \mathrm{~A}$, the center of the magnetic field is $0.1 \mathrm{~T}$. The external field magnet was assembled with the various supports to apply the parallel external magnetic field and perpendicular external magnetic field as shown in Fig. 4. (b), (c), respectively. Table II summarizes the specifications of the external magnetic field.

\section{CHARACTERISTICS EVALUATION OF THE HTS COILS UNDER EXTERNAL MAGNETIC FIELD}

3.1. Charge and discharge tests of the each HTS pancake coils without external magnetic field

Fig. 5 represents the operating current and Fig. 6, 7 represents central magnetic field according to the charge and discharge of the each HTS coil. The operating current varies from $10 \mathrm{~A}$ to $90 \mathrm{~A}$ at every $20 \mathrm{~A}$ and the rate of 


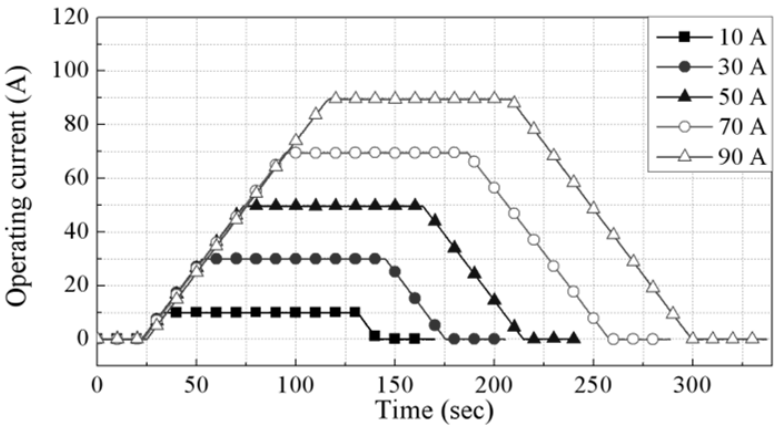

Fig. 5. Operating current test results of the HTS coils time function.

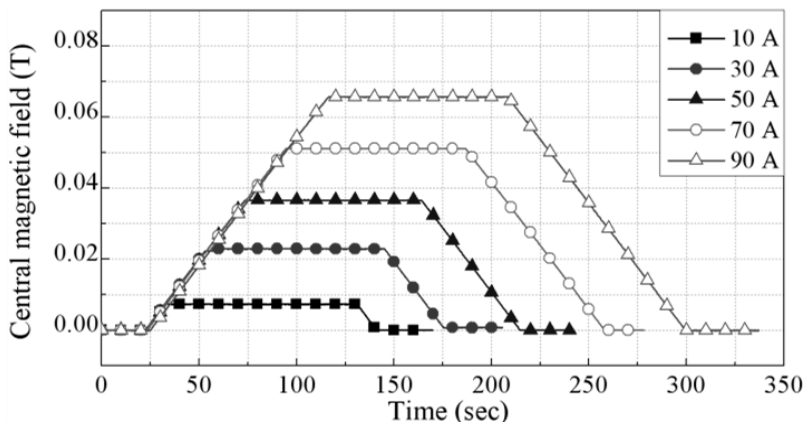

Fig. 6. Central magnetic field test results of the insulated HTS coil time function.

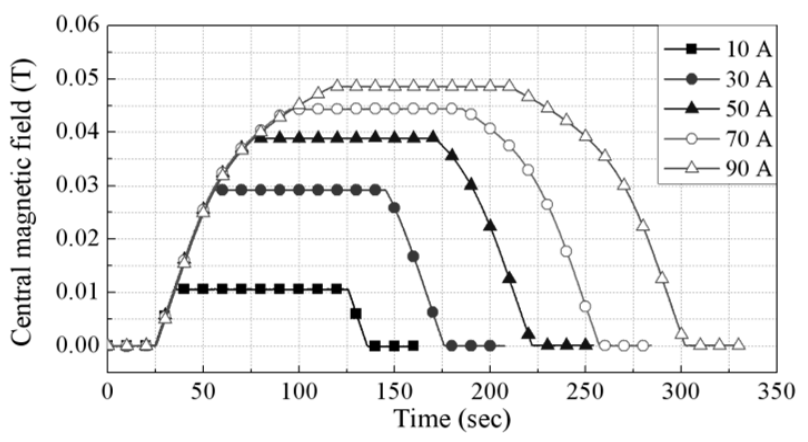

Fig. 7. Central magnetic field test results of the insulationless HTS coil time function.

charge and discharge is $1 \mathrm{~A} / \mathrm{s}$. The operating current increased up to each current level and kept at the level for $90 \mathrm{~s}$. In the insulated HTS coil, critical current was $23 \mathrm{~A}$, the central magnetic field increases linearly at each current level. With this, the normal charge and discharge are accomplished. But in the insulationless HTS coil, critical current was $23 \mathrm{~A}$, the central magnetic field is gradually saturated as 40,45 , and $49 \mathrm{mT}$ at 50, 70, and $90 \mathrm{~A}$ due to the current-sharing which was bypassed to another neighboring turn through turn-to-turn contact as the operating current exceed the critical current, as shown in Fig. 7.

3.2. Experiment results of the HTS coils under external magnetic field

Fig. 8 shows the decreasing level of the critical current of the each HTS coils under the different magnitude of

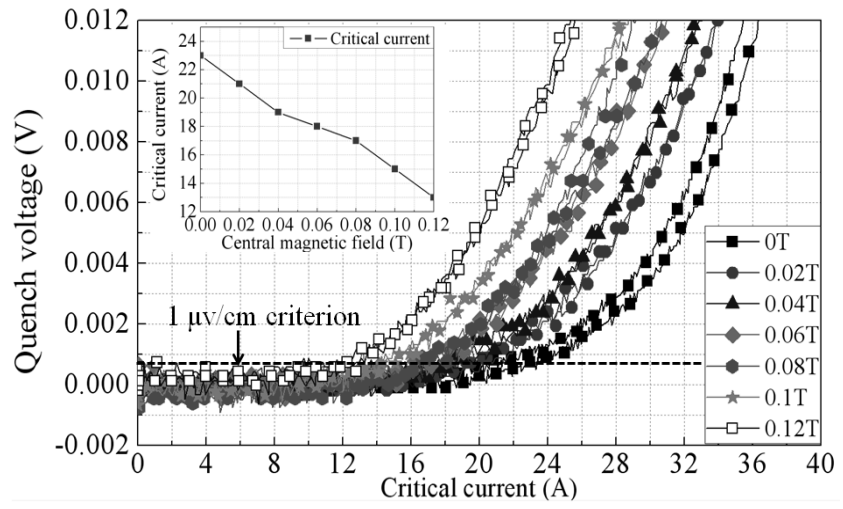

(a)

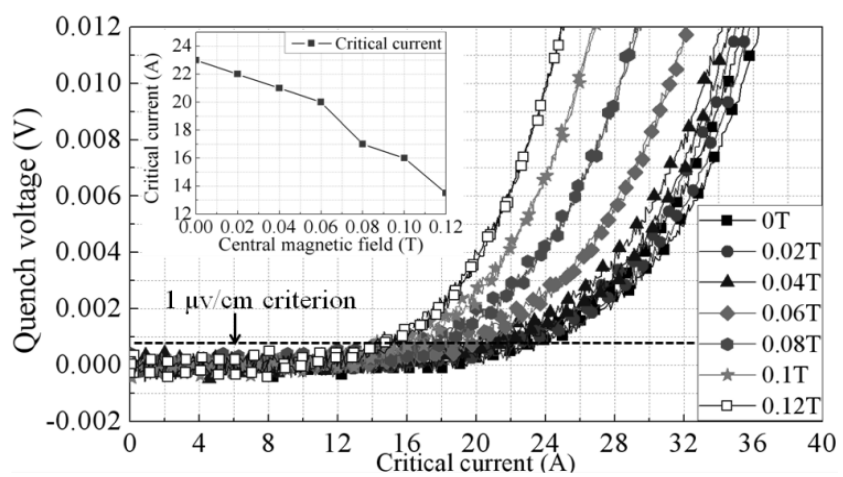

(b)

Fig. 8. Decreasing level of the critical current on the HTS coils under perpendicular external magnetic field: (a) insulated HTS coil, (b) insulationless HTS coil.

external magnetic field when applied the perpendicular magnetic field. The critical current of each HTS coil gradually decreased approximately $10 \mathrm{~A}$ When $0.02 \mathrm{~T}$ to $0.12 \mathrm{~T}$ external magnetic field applied, which presents different decreasing pattern, respectively, as depicted in Fig. 8 (a), (b). Consequently, these results prove that each HTS coils are influenced by external magnetic field and further quench would occur more quickly than conventional condition due to the decrease of critical current.

3.3. Over-current results of the HTS coils under external magnetic field

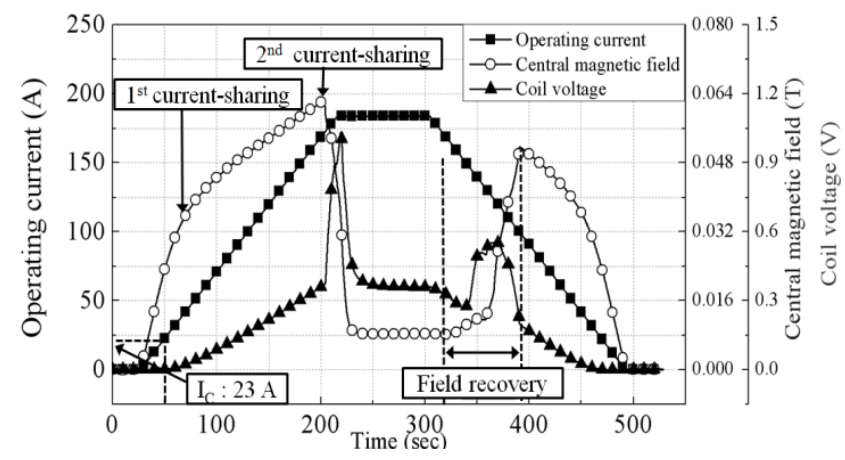

Fig. 9. Over-current test results of the insulationless HTS coil under $0.12 \mathrm{~T}$ external magnetic field. 


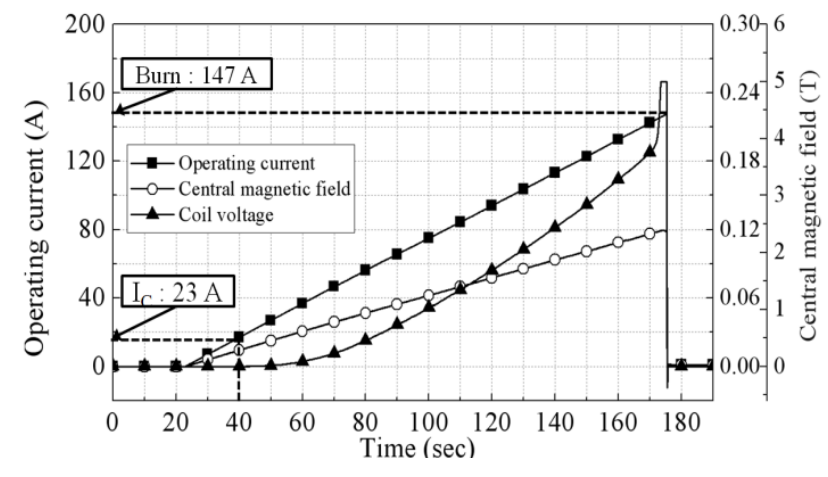

Fig. 10. Over-current test results of the insulated HTS coil under $0.12 \mathrm{~T}$ external magnetic field.

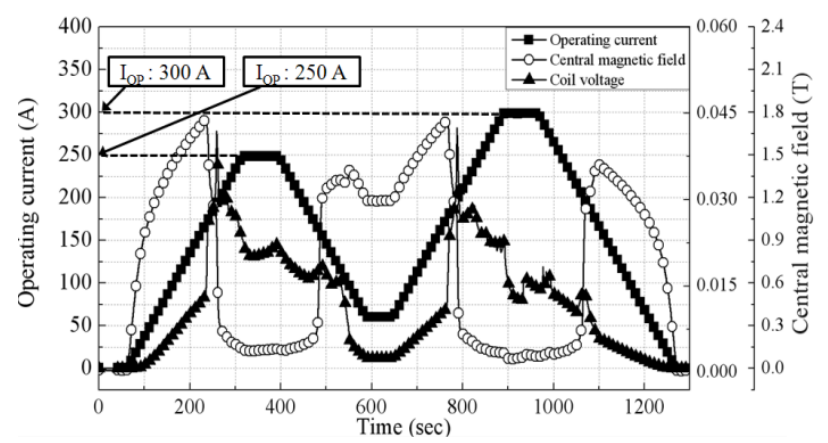

Fig. 11. Successive Over-current test results of the insulationless HTS coil under $0.12 \mathrm{~T}$ external magnetic field.

Fig. 9 represents the over-current test results of the insulationless HTS coil under $0.12 \mathrm{~T}$ external magnetic field. The square -marked line stands for the power supply current, the triangle -marked line shows the coil terminal voltage, and the circle-marked line represents the central magnetic field. At point " 1 st current-sharing", the inclination of the central magnetic field relieves because the current exceeds the critical current. At point " 2 nd current-sharing" the central magnetic field starts to decrease sharply. It means that the current reaches the critical current and then the primary current sharing occurs at the stabilizer layer of coil which changes the current density. So the inclination at " 2 nd current-sharing" was changed. For comparison, the insulated HTS coil test of the same conductor has been carried out, the test coil was

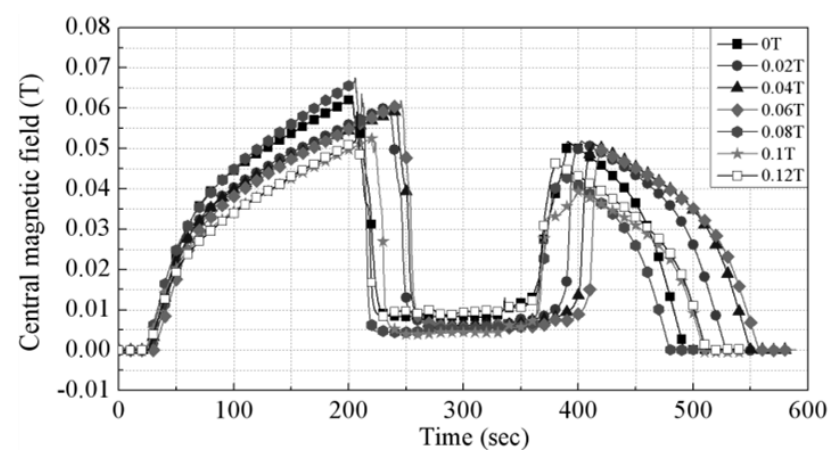

Fig. 12. Current-sharing characteristic of the insulationless HTS coil under external magnetic field. burned out at $147 \mathrm{~A}$, as depicted in Fig. 10. Fig. 11 shows the results of twice of charge and discharge for the insulationless HTS coil under $0.12 \mathrm{~T}$ external magnetic field. The primary charge current is $250 \mathrm{~A}$ and $300 \mathrm{~A}$ for the second charge. The insulationless HTS coil occurs current-sharing at the current higher than the critical current and then the characteristic of magnetic field recovered after the quench according to decrease of the operating current. It is confirmed that the insulationless HTS coil has better recovery characteristic against quench under external magnetic field compared with the insulated HTS coil.

3.4. Current-sharing characteristic of the insulationless HTS coil according to the external magnetic field.

Fig. 12 represents the current-sharing patterns of the insualtionless HTS coil according to the magnitude of external magnetic field in related to current-sharing characteristic. Patterns of the current-sharing occur irregularly over the critical current, as depicted in Fig. 10. From these results, the insulationless HTS coil wasn't damaged by the quench due to the current-sharing characteristic, although the critical current was decreased by the external magnetic field. However, an additional study on the correlation between critical current and current-sharing needs to be conducted.

\section{CONCLUSIONS}

The insulated and insulationless HTS coils were additionally tested for characteristics evaluation under the external magnetic field, which is dealt with in conventional papers. In the operating tests without external magnetic field, the insulationless HTS coil gradually saturated as exceeded the critical current due to current-sharing compared with the insulated HTS coil. When apply the external magnetic field, the critical current of each HTS coils decreases and current-sharing occurs irregularly over the critical current. Finally in the over-current tests, the insulationless HTS coil didn't burn out by quench due to current-sharing, while the insulated HTS coil burned out at 147 A under external magnetic field. These results prove that the insulationless HTS coil under the external magnetic field may guarantee better over-current stability than the insulated HTS coil.

\section{REFERENCES}

[1] D. Hazelton, V. Selvamanickam, J. Duval, D. Larbalestier, W. Markiewicz, H. Weijers, "Recent Developments in 2G HTS Coil Technology," IEEE Trans. Appl. Supercond., vol. 19, issue 3, pp. 2218-2222. 2009.

[2] D. Evans, "Turn, layer, and ground insulation for superconducting magnets," Physica C, issue 354, pp. 136-142, 2001.

[3] Y. Kim, S. Han, K. Kim, O. Kwon and H. Lee, "Investigation of HTS racetrack coil without turn-to-turn insulation for superconduting rotating machines," IEEE Trans. Appl. Supercond., vol. 22, issue 3, pp. 5200604, 2012.

[3] S. Hahn, D. Park, J. Bascunan and Y. Iwasa, "HTS Pancake Coils Without Turn-to-Turn Insulation," IEEE Trans. Appl. Supercond., vol. 21, issue 3, pp. 1592-1595, 2011.

[4] J. Lee, Y. Kwon, S. Baik, E. Lee, H. Kim, M. Park, I. Yu and Y. Jo, "Thermal Quench in HTS Double Pancake Race Track Coil," IEEE 
Trans. Appl. Supercond., vol. 17, issue 2, pp. 1603-1606, 2007.

[5] N. Akira, K. Hirohisa, and I, Takeshi, "An Experiment on Demagnetization of a Trapped Magnetic Field in HTS Bulk Exposed to an Alternating and Rotating Magnetic Field," IEEE Trans. Appl. Supercond., vol. 19, issue 3, pp. 3557-3560, 2009.

[6] S. Hidehito, U. Hiroshi, T. Makoto, and I. Atsushi, "Trapped Field
Characteristics of $\mathrm{Y}-\mathrm{Ba}-\mathrm{Cu}-\mathrm{O}$ Bulk in Time-Varying External Magnetic Field," IEEE Trans. Appl. Supercond., vol. 12, issue 1, pp. 820-823, 2002.

[7] M. Park, M. Choi, S. Hahn, G. Cha, and J. Lee, "Effect of the Stack in HTS Tapes Exposed to External Magnetic Field," IEEE Trans. Appl. Supercond., vol. 14, issue 2, pp. 1106-1109, 2004. 